\title{
Transient Optical Sky Survey Automated Telescope System
}

\author{
Elena Hadjiyska ${ }^{a}$, Philip Lubin ${ }^{a}$, Scott Taylor ${ }^{b}$, Gary Hughes ${ }^{c}$ \\ ${ }^{a}$ University of California at Santa Barbara; \\ ${ }^{\mathrm{b}}$ Raytheon Company; \\ ${ }^{\mathrm{c}}$ California Polytechnic State University, San Luis Obispo
}

\begin{abstract}
We describe the optical design of a sky survey system comprised of small aperture telescope tube assemblies mounted on a common semi-equatorial frame with a single polar axis. It is the first ground-based instrument to create a map of transients down to optical $m=17$ by imaging a fixed-declination strip of the sky on a nightly basis. The system is fully remotely automated and physically robust. The mount tracks the sky using a motion controller, drive motor, and a laser rotary encoder. The prototype configuration is suited to house up to 6 telescopes on the current mount and is easily expandable to accommodate up to 30 telescopes which would enable full sky coverage if one system each were placed in the Northern and Southern Hemispheres ${ }^{1}$.
\end{abstract}

Keywords: sky survey, small aperture telescope, transients

\section{TOSS OVERVIEW}

The Transient Optical Sky Survey (TOSS) is a system of optical telescopes designed to survey the sky to a limit of 17th magnitude, and to catalog transients by analyzing time-series luminosity measurements of detected objects from nightly observations $^{2}$. We describe the optical system, focal plane cameras, and electromechanical pointing system used to achieve the science objective. The proof of concept system currently consists of two telescopes, as shown in Figure 1.

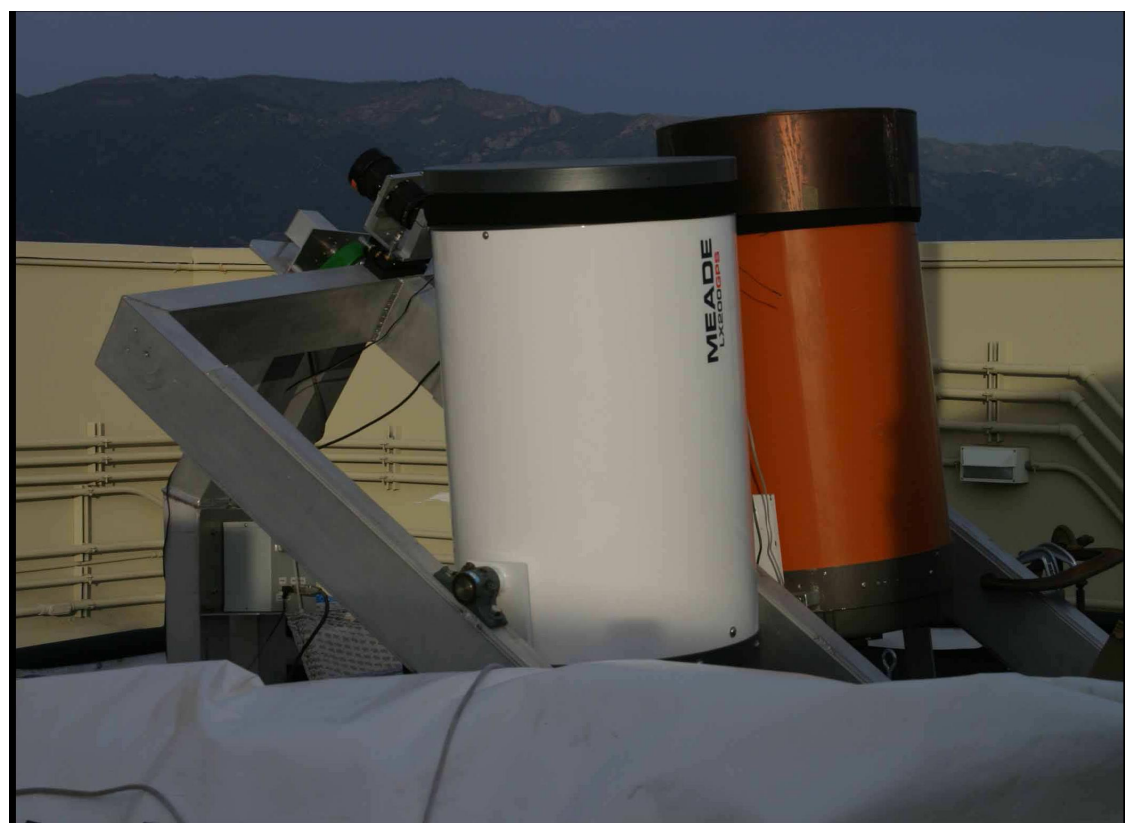

Fig. 1. Celestron (14 inch aperture, f/11) and MEADE (16 inch aperture, f/11), on aluminum mount. 


\section{CHARACTERISTICS}

Successful operation of TOSS required integration of several subsystems, including the cameras, telescopes, optical hardware, and mechanical motion control elements. The performance specifications of these components is listed in Table 1. In addition, several items not listed aid in the calibration and data collection during various weather conditions. These items include a polar alignment telescope, electronic focusers, dew shields, and dew heaters.

Table 1. Characteristics of the TOSS system and its components.

\begin{tabular}{|c|c|c|c|}
\hline Parameter & Value & Units & Notes \\
\hline \multicolumn{4}{|c|}{ TOSS System } \\
\hline Limiting Magnitude & 18 & Mag & \\
\hline RA Field & 1800 & $\operatorname{arcsec}$ & Per camera \\
\hline Dec Field & 1200 & $\operatorname{arcsec}$ & Per camera \\
\hline \multicolumn{4}{|c|}{ Cameras } \\
\hline Tint & 120 & $\mathrm{sec}$ & Integration time \\
\hline Plate scale & 0.667 & arcsec/pixel & \\
\hline Pixel size & $7.4 \times 7.4$ & $\mathrm{~m}^{2}$ & 3 colors \\
\hline Camera Format & $3072 \times 2048$ & pixels & \\
\hline Read Noise & 14 & e- & \\
\hline Well size & 250000 & e- & \\
\hline Gain & 4.0 & e-/ADU & \\
\hline Resolution & 16 & Bits & Per color \\
\hline Filter & RGB Bayer & & \\
\hline \multicolumn{4}{|c|}{ Meade Telescope } \\
\hline Optical System & Catadioptric & & \\
\hline Aperture & 40.64 & $\mathrm{~cm}$ & \\
\hline Focal Length & 406.4 & $\mathrm{~cm}$ & \\
\hline \multicolumn{4}{|c|}{ Celestron Telescope } \\
\hline Optical System & Catadioptric & & \\
\hline Aperture & 35.56 & $\mathrm{~cm}$ & \\
\hline Focal Length & 355.6 & $\mathrm{~cm}$ & \\
\hline \multicolumn{4}{|c|}{ Focal Length Reducer } \\
\hline Reduction & 0.63 & & \\
\hline \multicolumn{4}{|c|}{ Mechanical System } \\
\hline Pointing Stability & \pm 1 & $\operatorname{arcsec}$ & Over $120 \mathrm{sec}$ integration time \\
\hline Range of motion & \pm 10 & degree & Allows for calibration \\
\hline
\end{tabular}

\section{SYSTEM BLOCK DIAGRAM}

The telescopes are mounted on a robust aluminum frame which is free to rotate in the right ascension direction only. Its RA axis is fixed to a horizontal triangular frame, which has a jackscrew in each corner. The three jackscrews allow precise alignment to the celestial axis. Both telescopes are pointed at the zenith, and offset from each other in declination. To achieve the pointing stability required, an electromechanical system is used. It consists of a Galil motor controller, DC motor with encoder, and an optical encoder with a sine wave interpolator. The system's position encoder has a resolution of $0.5 \mathrm{arcsec}$. Setting stable values of position, integral and derivative allows accurate tracking through the $120 \mathrm{sec}$ integration time. The Galil is also used to control the exposure time of the cameras. A computer controls the system, which is shown in Figure 2. 


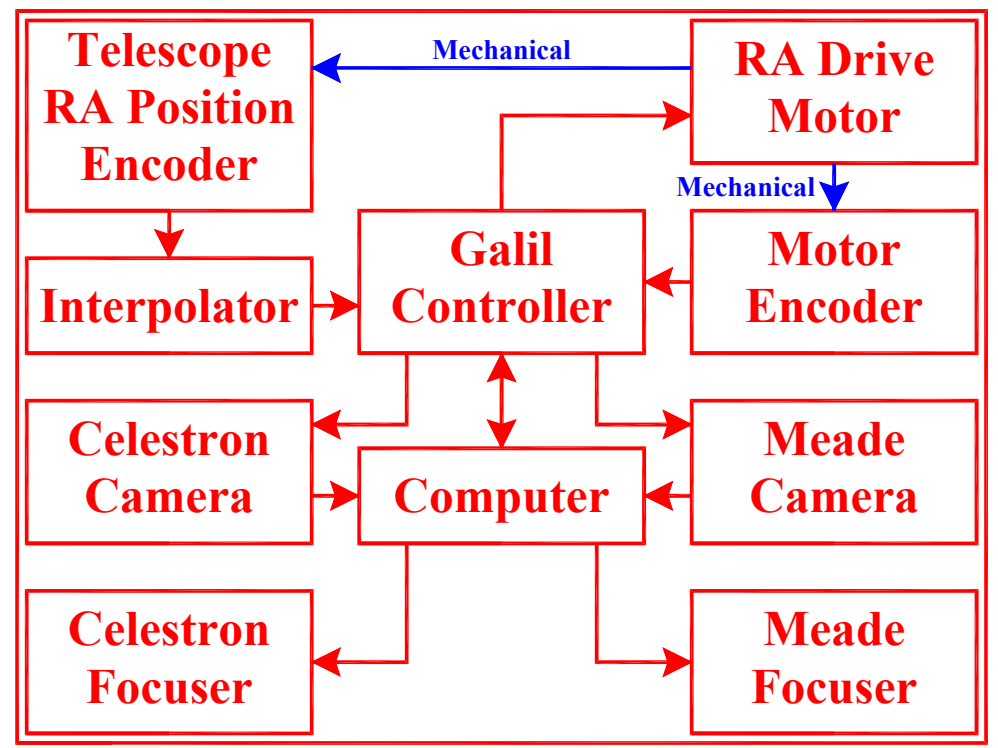

Fig. 2. TOSS electromechanical system.

\section{OPTICAL SYSTEM}

The optical system is shown in Figure 3. It consists of a Meade 16" aperture telescope and a Celestron 14" telescope, followed by a focal length reducer, and an automatic focuser. The camera is mounted to the focuser. A dew shield is used to reduce the condensation of dew and to block stray light from entering the telescope. In addition, a dew heater maintains the temperature of the corrector plate at just above the dew point. This allows optimum performance in the often high relative humidity found on site. The focuser is designed to compensate for its own thermal expansion and contraction with temperature, and it has additional control to eliminate temperature effects on focus from the rest of the system. The focal length reducer allows optimization of the focal length of the telescope to the pixel size of the camera.

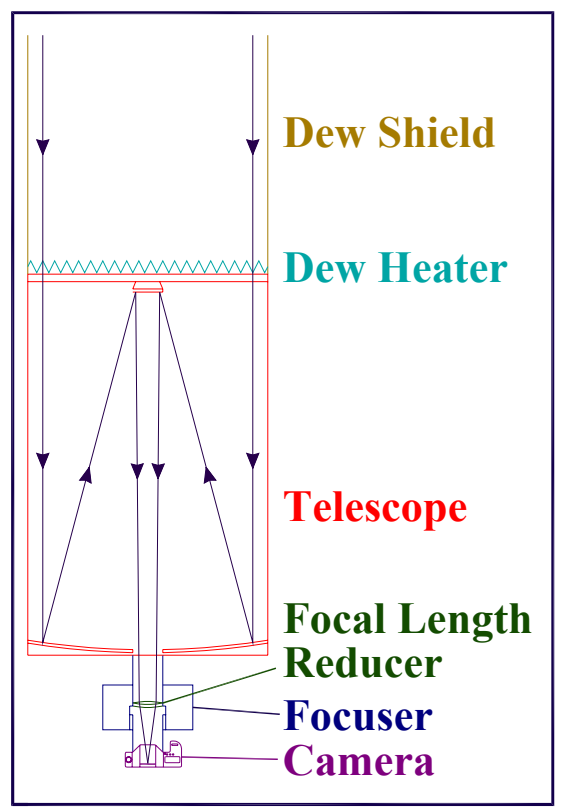

Fig. 3. TOSS optical system. 


\section{CAMERA READ NOISE AND GAIN}

The read noise and gain of both cameras were measured with a uniform, constant intensity light source, and varying the integration time. The variance was plotted against the standard deviation of the central $100 \mathrm{X} 100$ pixels. The slope of the resulting data produces the gain in electrons/ADU, and the zero intercept is the read noise in electrons. This is shown in Figure 4. The noise and gain of the two cameras is listed in Table 2. Both cameras were at 22C temperature, and the ISO was set to 400 . The ISO sets the relative gain on digital cameras.

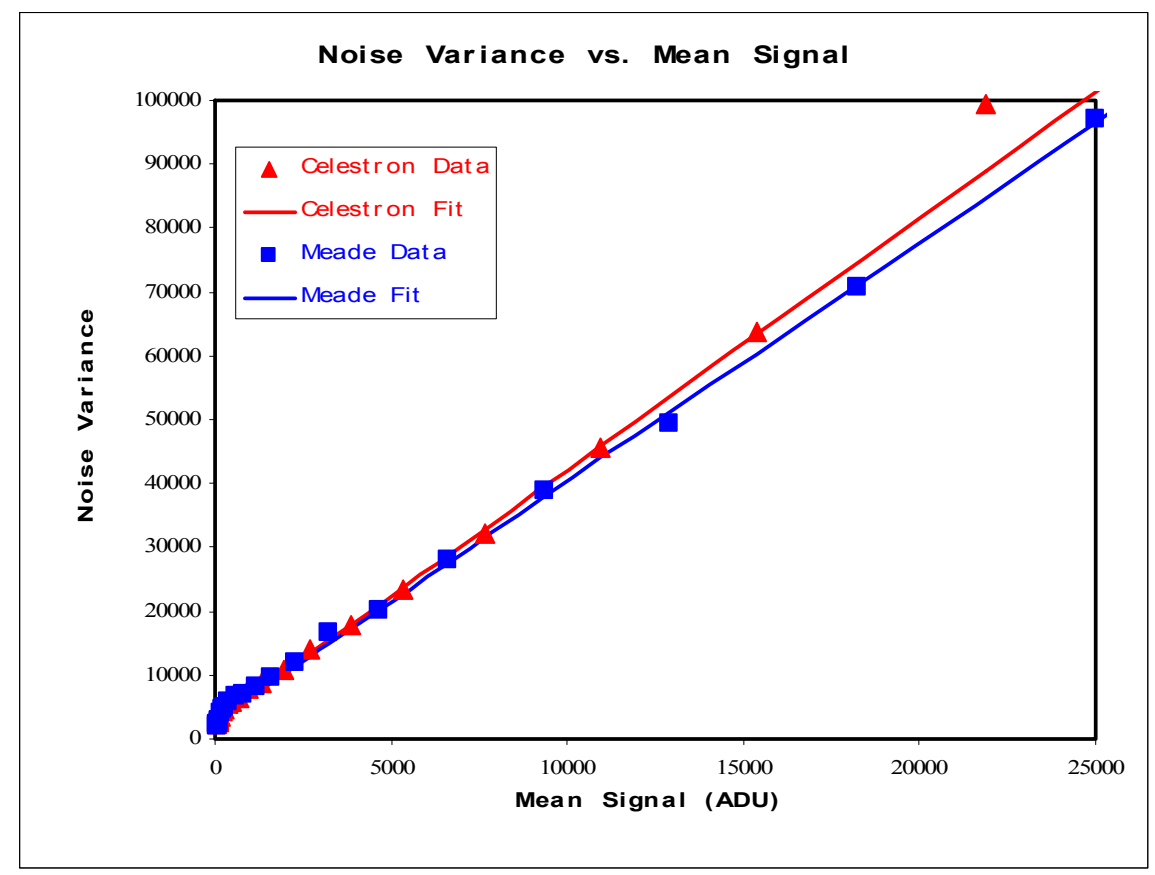

Fig. 4. Noise variance vs. mean signal plot for the two cameras..

Table 2. Noise and gain for the two cameras.

\begin{tabular}{|l|c|r|}
\hline \multicolumn{1}{|c|}{ Parameter } & Celestron & Meade \\
\hline Noise (e-) & 13.17 & 14.18 \\
\hline Gain (e-/ADU) & 3.94 & 3.75 \\
\hline
\end{tabular}




\section{CAMERA SPECTRAL RESPONSE}

The spectral response of each camera was measured using an Optronics spectrometer. Data was taken every 10nm from $350 \mathrm{~nm}$ to $1200 \mathrm{~nm}$. A standard detector with calibration traceable to NIST was used to determine relative spectral quantum efficiency. The relative spectral quantum efficiency for the Meade and Celestron cameras is shown in Figure 5.

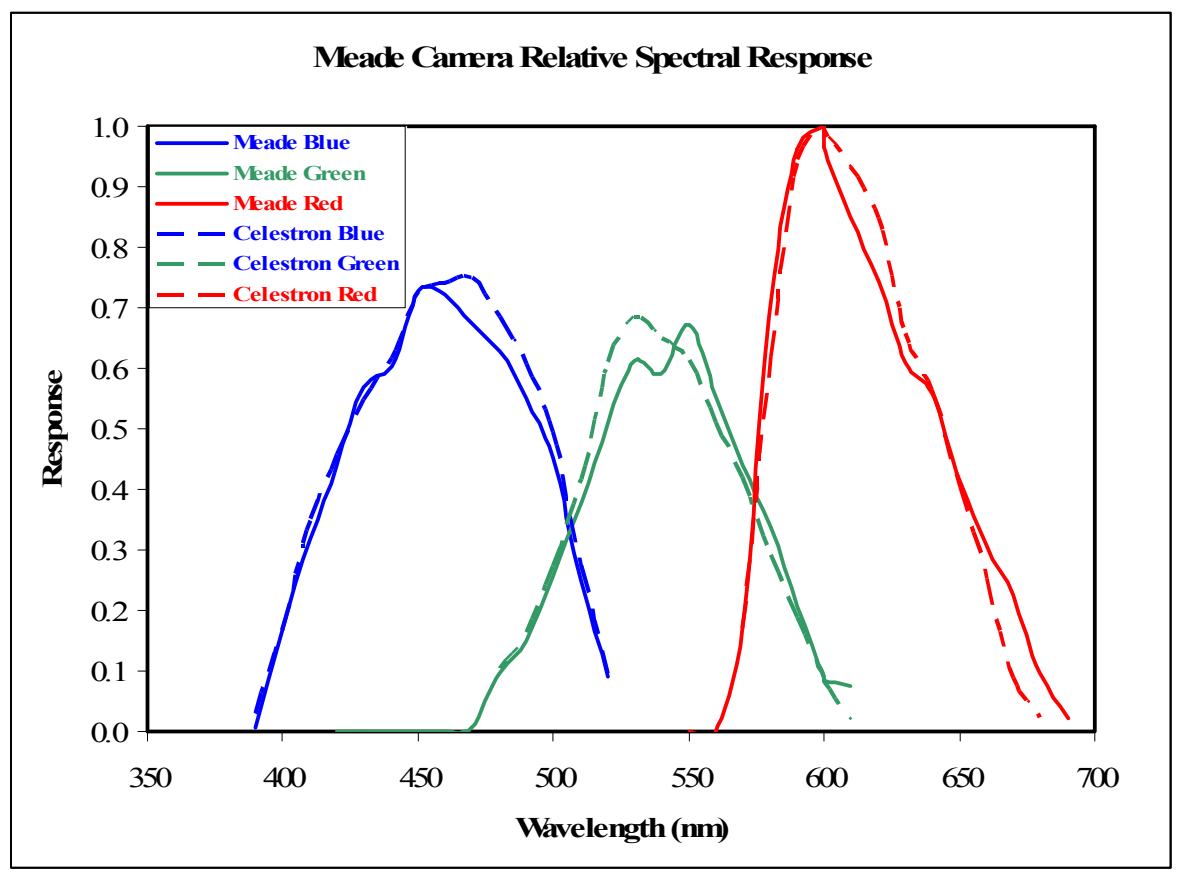

Fig. 5. The relative spectral quantum efficiency for each color for the Meade and Celestron cameras.

\section{DARK FRAME AND FLAT FIELD CHARACTERIZATION}

The dark frame corrects for camera array anomalies, while the flat field corrects for optical deformations. The dark frame is shown in Figure 6, and the flat field is shown in Figure 7. The dark-frame offset is just a few hundred counts out of 65535 maximum counts. The dark field data was taken at each of seven temperatures for each camera. Sixteen frames were taken and averaged at each temperature, to reduce the dark field noise contribution when subtracted from the image frames. All data was taken with $120 \mathrm{sec}$ exposure. The dark field subtraction also eliminates "hot" pixels from the resulting image. An average of sixteen dark field exposures is shown in Figure 6. 


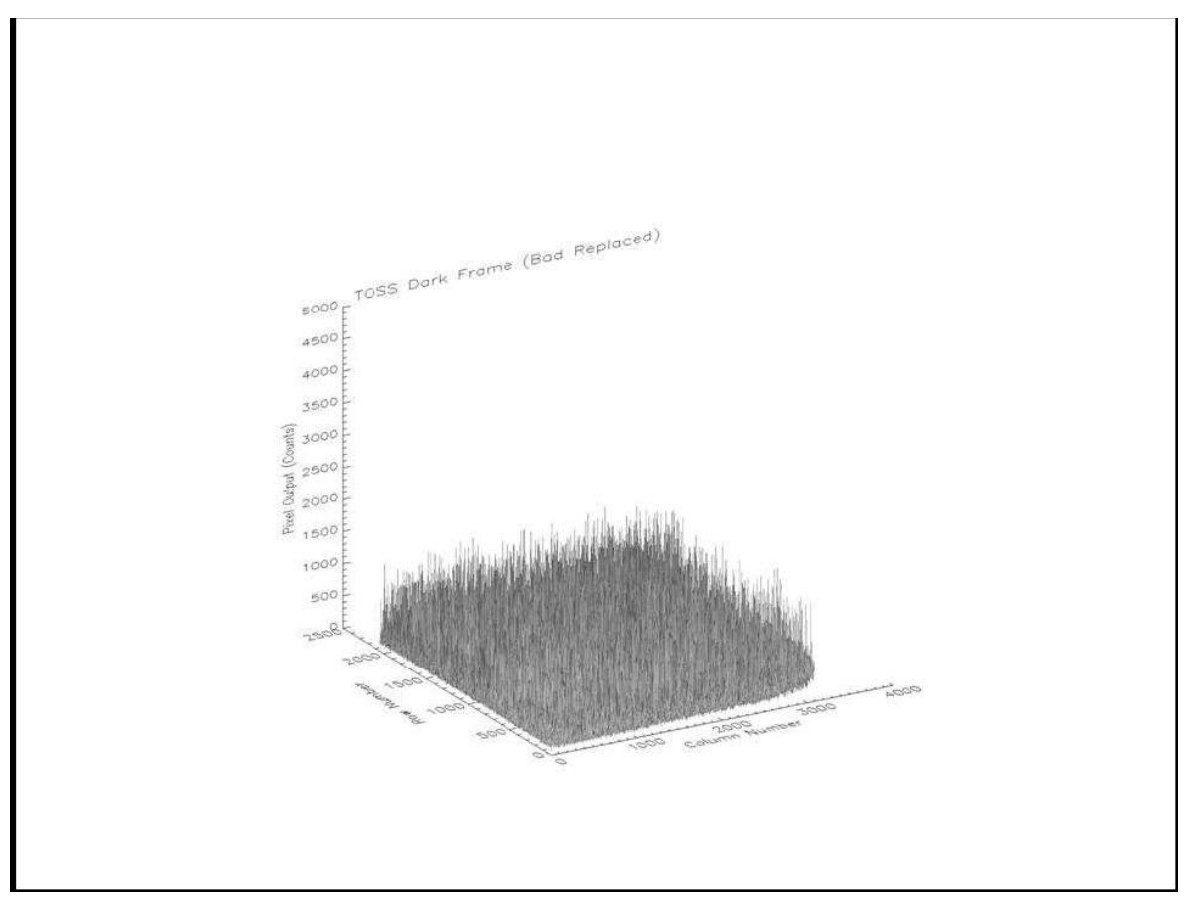

Fig. 6. Dark field data for the Meade camera.

The flat field frame is used to correct for the optical effects in the system. The image data is divided by this frame to correct the gain, which varies over the field of the camera mainly because of the focal length reducer. A flat field frame is shown in Figure 7.

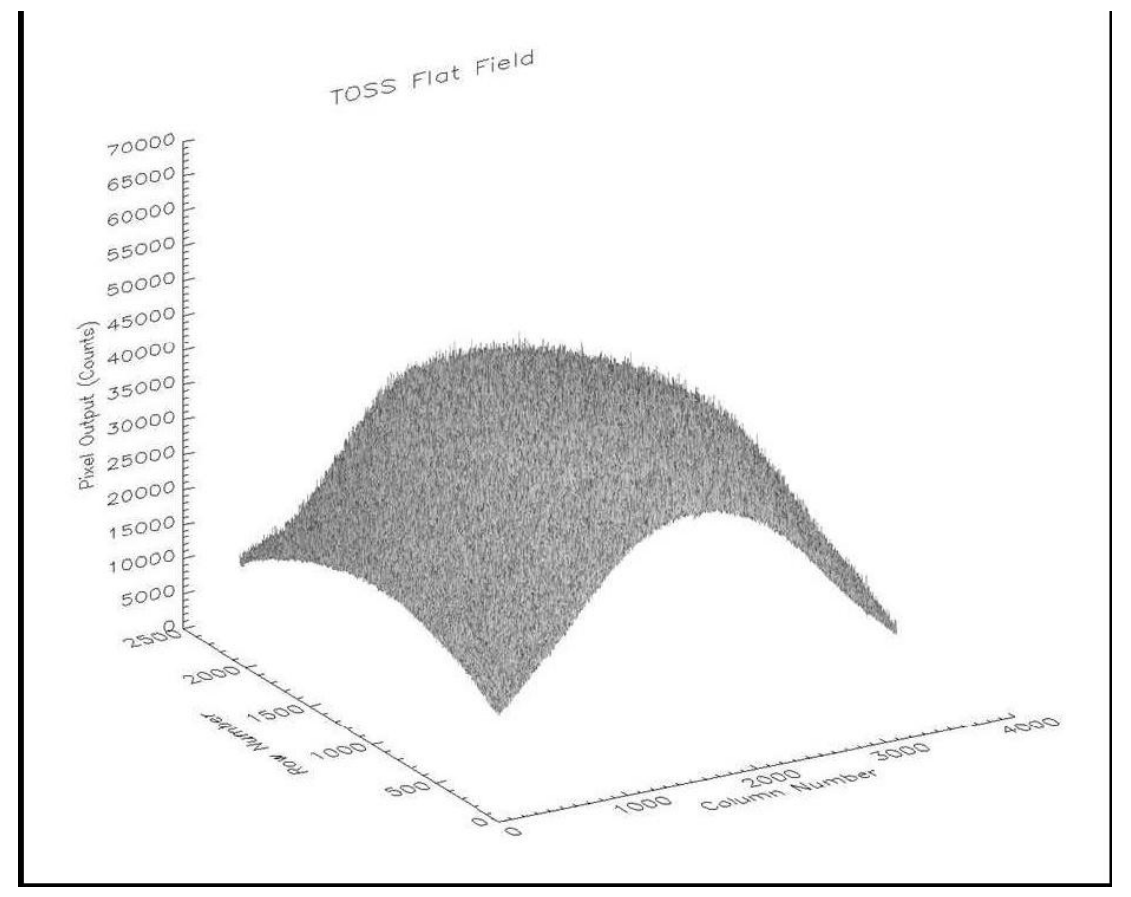

Fig. 7. Flat field data for the Meade camera. 


\section{LIMITING MAGNITUDE CALCULATION}

The limiting magnitude achievable with the TOSS system was calculated using data from a known star. After allowing for the air mass, less than ideal focus of the telescope, and the short integration time (30 sec vs. $120 \mathrm{sec}$ ), the limiting star magnitude is at least 17.5. The seeing conditions were average when the data was taken. A signal to noise ratio of 1.5 was assumed, as this is the lower limit at which the analysis software can reliably detect objects. The calculations are shown in Table 3.

Table 3. Limiting magnitude calculation.

\begin{tabular}{|l|c|c|c|}
\hline \multicolumn{1}{|c|}{ Star } & GSC 1266:11 & Limit Star & Unit \\
\hline Magnitude & 10.28 & 17.53 & Mag \\
\hline Exposure Time & 30 & 120 & sec \\
\hline ISO & 400 & 400 & ratio \\
\hline Air Mass & 1.06 & 1.00 & ratio \\
\hline FWHM X & 9.9 & 3.0 & pixel \\
\hline FWHM Y & 6.1 & 3.0 & pixel \\
\hline Noise Floor & 77 & 154 & e- \\
\hline Signal & 6101.22 & 231 & ADU \\
\hline
\end{tabular}

\section{DEW SHIELD CALCULATION}

Dew shields provide multiple advantages. Most importantly, the dew shield reduces the tendency for the telescope optics to reach thermal equilibrium with the air above it. Since our telescopes are pointed at the zenith, the optics attempts to reach a temperature of $-40 \mathrm{C}$, which is the average temperature of the air above us. Long before the optics reach that low temperature, however, condensation of moisture in the air occurs on the corrector plate. This limits the cooling process, but it makes the telescope unusable. A dew shield works by limiting the thermal field of view of the optics (which is much greater than the optical field of view), and thus limiting the amount of heat the optics can radiate to the air above. The solid angle and reduction in heat loss for our telescope are shown in Figure 8. A dew heater with a thermocouple temperature feedback provides additional heat to further prevent condensation.

The dew shield also greatly reduces stray light from entering the optical path of the telescope, and it reduces the collection of dust and contaminants on the collector plate. The dew shield was constructed of aluminum sheet with optically absorbing black felt lining its inside surface. 


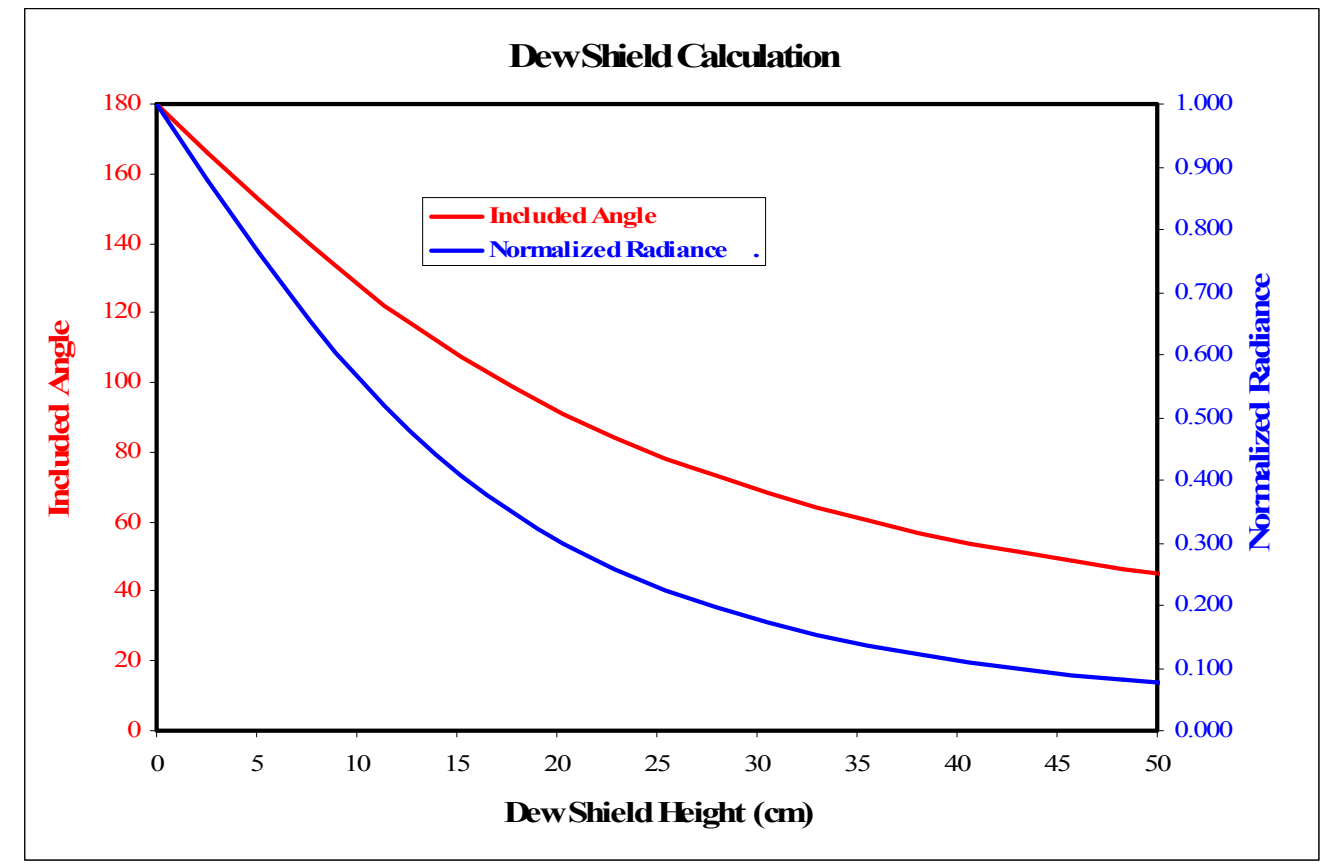

Fig. 8. Dew shield included angle and normalized radiance at the corrector plate vs. height in $\mathrm{cm}$.

\section{CONCLUSION}

The Transient Optical Sky Survey system was developed to provide a low cost, automated solution to conducting an extensive search for transients. A large number of such systems could result in a very substantial quantity of transients reported in a timely manner. Accurate calibration of a commercially available digital camera, coupled with the long exposure times possible, greatly extends the magnitude range possible.

\section{REFERENCES}

[1] Hadjiyska, E., Lubin, P., Taylor, S., Zierten, J., Riley, A., O’Neill, H., Ansmann, M., Leonardi, R., Rubin, I., Newton, E., Eidman, C., Cole, T., Davis, V., Finney, J., Moshman, N., Quetin, E., Meinhold, P., Mac Moore, I. , " Transient Optical Sky Survey," American Astronomical Society Meeting 210 poster, (2007).

[2] Marvil, J., Ansmann, M., Childers, J., Cole, T., Davis, G.V., Hadjiyska, E., Halevi, D., Heimberg, G., Kangas, M., Levy, A., Leonardi, R., Lubin, P., Meinhold, P., O'Neill, H., Parendo, S., Quetin, E., Stebor, N., Villela, T., Williams, B., Wuensche, C.A., Yamaguchi, K.., " An Astronomical Site Survey at the Barcroft Facility of the White Mountain Research Station," New Astronomy, 11, (2006). 